\title{
THE BLOW UP ANALYSIS OF THE GENERAL CURVE SHORTENING FLOW
}

\author{
RONGLI HUANG ${ }^{1,2}$ AND JIGUANG BAO ${ }^{3}$
}

\begin{abstract}
It is shown that the curvature function satisfies a nonlinear evolution equation under the general curve shortening flow and a detailed asymptotic behavior of the closed curves is presented when they contract to a point in finite time.
\end{abstract}

\section{INTRODUCTION}

The curve shortening flows have been studied by many authors and have many applications (cf. [1]). M.E.Gage and R.S.Hamilton discussed the mean curvature flow in one dimensional case (cf. [2]). The flow is given by the equation

$$
v=k,
$$

where $v$ and $k$ are, respectively, the normal velocity and inward curvature of the plane curve. They proved that a convex closed curve stays convex and smooth and shrinks to a point in finite time with the limiting shape of a circle. In this paper, we generalized some results on curve shortening flow (cf. [2]) to the generalized curve shortening flow (cf. [3])

$$
v=|k|^{p-1} k
$$

where $p$ is the positive number. When $p=\frac{1}{3}$, the flow (1.1) is the affine plane curve evolution (cf. [4], [5]). More generally, we will also study the non-homogeneous flow (cf. [3]) which is given by

$$
v=G(k) k,
$$

where $G(\cdot)$ is a positive function on $(0, \infty)$. Chia-Hsing Nien and Dong-Ho Tsai had proved that the self-similar solutions under the contraction flow could happen only as (1.1) (cf. [6]). B.Andrews had studied some nonlinear expansion of contraction flow and obtained the limiting self-similar solutions (cf. [3]). But the asymptotic behavior of solutions of the flow (1.1), (1.2) are little known. The main objective of this paper is to analyze the asymptotic behavior of the curvature under the generalized curve shortening flow .

2000 Mathematics Subject Classification. 35K45; 35K65 .

Key words and phrases. Asymptotic behavior, Curve shortening flow, Support function, Hausdorff metric. 
Let $\mathbb{S}^{1}$ be an unit circle in the plane, and

$$
\gamma_{0}: \mathbb{S}^{1} \rightarrow \mathbb{R}^{2}
$$

be closed convex curve in the plane. We look for a family of closed curves

$$
\gamma(u, t): \mathbb{S}^{1} \times[0, T) \rightarrow \mathbb{R}^{2}
$$

which satisfies

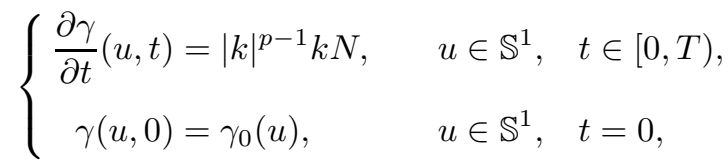

where $p$ is a positive number, $k(\cdot, t)$ is the inward curvature of the plane curve $\gamma(\cdot, t)$ and $N(\cdot, t)$ is the unit inward normal vector. More generally, we consider $\gamma(\cdot, t)$ satisfying

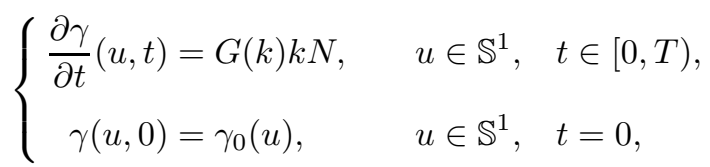

where $G$ is a positive, non-decreasing smooth function on $(0, \infty)$.

In the following sections we assume that $A(t)$ is the area of a bounded domain enclosed by the curve $\gamma(\cdot, t), L(t)$ is the length of $\gamma(\cdot, t), r_{\text {out }}(t)$ and $r_{\text {in }}(t)$ are respectively the radii of the largest circumscribed circle and the smallest circumscribed circle of $\gamma(\cdot, t)$. Define

$$
\begin{aligned}
& k_{\max }(t)=\max \left\{k(u, t) \mid u \in \mathbb{S}^{1}\right\}, \\
& k_{\min }(t)=\min \left\{k(u, t) \mid u \in \mathbb{S}^{1}\right\} .
\end{aligned}
$$

Firstly we introduce the existence theorem, which belongs to Ben.Andrews (cf. Theorem П4.1, Proposition $\Pi 4.4$ in [3]).

Proposition 1.1. Let $\gamma_{0}$ be a closed strictly convex curve. Then the unique classical solution $\gamma(\cdot, t)$ of (1.3) exists only at finite time interval $[0, \omega)$, and the solution $\gamma(\cdot, t)$ converges to a point $\vartheta$ as $t \rightarrow \omega$ and $A(t), k_{\max }(t)$ satisfy the following properties:

$$
\begin{aligned}
& \forall t \in[0, \omega), A(t)>0, k_{\max }(t)<+\infty, \\
& \lim _{t \rightarrow \omega} A(t)=0, \quad \lim _{t \rightarrow \omega} k_{\max }(t)=+\infty .
\end{aligned}
$$

As $t \rightarrow \omega$, the normalized curves

$$
\eta(\cdot, t)=\sqrt{\frac{\pi}{A(t)}} \gamma(\cdot, t)
$$

converges to the unit circle centered at the point $\vartheta$. 
In this paper we always assume that the initial curve satisfies the conditions of Proposition 1.1 . and that $G(x)$ is a function on $(0, \infty)$ satisfying

(H1) $G(x) \in C^{3}(0, \infty), G^{\prime}(x) \geq 0$ and $G(x)>0$ for $x \in(0, \infty)$.

(H2) $G(x) x^{2}$ is convex in $(0, \infty)$ and there is a positive constant $C_{0}$ such that

$$
G^{\prime}(x) x \leq C_{0} G(x), \text { for sufficiently large } x .
$$

We now state the main theorem of this paper.

Theorem 1.2. Suppose $G(x)$ satisfies $(H 1)$ and $(H 2)$. Let $\gamma(\cdot, t)$ be the solution for Proposition

Then the following hold:

i) $\lim _{t \rightarrow \omega} \frac{r_{\text {in }}(t)}{r_{\text {out }}(t)}=1$.

ii) $\lim _{t \rightarrow \omega} \frac{k_{\min }(t)}{k_{\max }(t)}=1$.

iii) $\lim _{t \rightarrow \omega} \frac{1}{\omega-t} \int_{k(\theta, t)}^{+\infty} \frac{d x}{G(x) x^{3}}=1$ is uniformly convergent on $\mathbb{S}^{1}$.

Remark 1.3. Let $G(x)=|x|^{p-1}$ with $p \geq 1$ in Proposition 1.1. Then

$$
k(\theta, t)[(p+1)(\omega-t)]^{\frac{1}{p+1}} \text { converges uniformly to } 1 \text { as } t \rightarrow \omega .
$$

for uniformly $\theta$ in $\mathbb{S}^{1}$.

Remark 1.4. When $p=1$, by 1.4) it shows that the asymptotic formula about curvature function of the curve shortening flow (1.1) which was discovered firstly by M.Gage and R.S.Hamilton (cf. Corollary 5.6 in [2]).

This paper is organized as follows: In the next section we transfer the flow (1.3) into an initial PDEs problem and establish some monotone geometric inequality. Section 3 is devoted to the main contribution of our article, that is, the global Harnack inequality of the curvature function according to the flow (1.3), see Lemma 3.6. And then we complete the proof of Theorem 1.2 by making use of Gage-Hamilton's methods (cf. [2]).

\section{Evolutions}

Using the idea in [2], we can drive the evolution equations under the flow (1.3) for the length and the curvature of the curves, and the area enclosed by the curves.

Let the curve be $\gamma(u)=(x(u), y(u))$ with parameter $u$ (modulo $2 \pi$ ) and $s$ be an arc-length parameter along the curve $\gamma(u)$ which is unique up to a constant. Then

$$
d s=v d u, \quad \frac{\partial}{\partial s}=\frac{1}{v} \frac{\partial}{\partial u}
$$

where

$$
v=\sqrt{\left(\frac{\partial x}{\partial u}\right)^{2}+\left(\frac{\partial y}{\partial u}\right)^{2}}
$$


Suppose $\xi$ and $N$ are the unit tangent vector and the unit inward normal vector of the curve. Then the Frenet equations (cf. [7]) are

$$
\frac{\partial \xi}{\partial u}=v k N, \quad \frac{\partial N}{\partial u}=-v k \xi
$$

or

$$
\frac{\partial \xi}{\partial s}=k N, \quad \frac{\partial N}{\partial s}=-k \xi
$$

where $k(u)$ is the inward curvature of $\gamma(u)$.

Let $\theta$ be the tangent angle of the curve $\gamma(u)$ to the $x$-axis. We drive the following useful formula.

Lemma 2.1. The geometric quantities $v, L, \zeta, N, \theta$ and $k$ of the flow (1.3) evolve according to
i) $\frac{\partial v}{\partial t}=-G(k) k^{2} v$,
ii) $\frac{d L}{d t}=-\int_{0}^{L} G(k) k^{2} d s$,
iii) $\frac{\partial}{\partial t} \frac{\partial}{\partial s}=\frac{\partial}{\partial t} \frac{\partial}{\partial s}+G(k) k^{2} \frac{\partial}{\partial s}$,
iv) $\frac{\partial \xi}{\partial t}=(G(k) k)^{\prime} \frac{\partial k}{\partial s} N, \frac{\partial N}{\partial t}=-(G(k) k)^{\prime} \frac{\partial k}{\partial s} \xi$,
v) $\frac{\partial \theta}{\partial t}=(G(k) k)^{\prime} \frac{\partial k}{\partial s}, \frac{\partial \theta}{\partial s}=k$, where $\xi=(\cos \theta, \sin \theta)$,
vi) $\frac{d A}{d t}=-\int_{0}^{L} G(k) k d s$
vii) $\frac{\partial k}{\partial t}=\frac{\partial}{\partial s}\left((G(k) k)^{\prime} \frac{\partial k}{\partial s}\right)+G(k) k^{3}$.

Proof. Let $\langle\cdot, \cdot\rangle$ be the inner product in $\mathbb{R}^{2}$.

i) By (1.3) and the Frenet equations we have

$$
\begin{aligned}
\frac{\partial}{\partial t}\left(v^{2}\right) & =\frac{\partial}{\partial t}\left\langle\frac{\partial \gamma}{\partial \omega}, \frac{\partial \gamma}{\partial \omega}\right\rangle \\
& =2\left\langle\frac{\partial \gamma}{\partial \omega}, \frac{\partial^{2} \gamma}{\partial \omega \partial t}\right\rangle \\
& =2\left\langle v \xi, \frac{\partial}{\partial \omega}(G(k) k N)\right\rangle \\
& =2\left\langle v \xi, \frac{\partial}{\partial \omega}(G(k) k) N+G(k) k \frac{\partial N}{\partial \omega}\right\rangle \\
& =2\left\langle v \xi, \frac{\partial}{\partial \omega}(G(k) k) N-G(k) k^{2} v \xi\right\rangle \\
& =-2 G(k) k^{2} v^{2} .
\end{aligned}
$$

This implies that the identity (i) holds.

ii) Since $L=\int_{0}^{2 \pi} v d \omega$, then from (i) there holds

$$
\frac{\partial L}{\partial t}=\int_{0}^{2 \pi} \frac{\partial v}{\partial t} d \omega=-\int_{0}^{2 \pi} G(k) k^{2} v d \omega=-\int_{0}^{L} G(k) k^{2} d s .
$$


iii) By (i) we get

$$
\begin{aligned}
\frac{\partial}{\partial t} \frac{\partial}{\partial s} & =\frac{\partial}{\partial t}\left(\frac{1}{v} \frac{\partial}{\partial \omega}\right) \\
& =-\frac{1}{v^{2}} \frac{\partial v}{\partial t} \frac{\partial}{\partial \omega}+\frac{1}{v} \frac{\partial}{\partial t} \frac{\partial}{\partial \omega} \\
& =-\frac{1}{v^{2}}\left(-G(k) k^{2} v\right) \frac{\partial}{\partial \omega}+\frac{\partial}{\partial s} \frac{\partial}{\partial t} \\
& =G(k) k^{2} \frac{\partial}{\partial s}+\frac{\partial}{\partial s} \frac{\partial}{\partial t}
\end{aligned}
$$

iv) It follows from (1.3) and (iii) that

$$
\frac{\partial \xi}{\partial t}=\frac{\partial}{\partial t} \frac{\partial}{\partial s} \gamma=\frac{\partial}{\partial s} \frac{\partial}{\partial t} \gamma+G(k) k^{2} \frac{\partial}{\partial s} \gamma=\frac{\partial}{\partial s}(G(k) k N)+G(k) k^{2} \xi
$$

Then by Frenet equations we have

$$
\begin{aligned}
\frac{\partial \xi}{\partial t} & =\frac{\partial}{\partial s}(G(k) k) N+G(k) k \frac{\partial N}{\partial s}+G(k) k^{2} \xi \\
& =(G(k) k)^{\prime} \frac{\partial k}{\partial s} N-G(k) k^{2} \xi+G(k) k^{2} \xi \\
& =(G(k) k)^{\prime} \frac{\partial k}{\partial s} N .
\end{aligned}
$$

In terms of $\langle\xi, N\rangle \equiv 0$ we obtain

$$
0 \equiv \frac{\partial}{\partial t}\langle\xi, N\rangle=\left\langle\frac{\partial \xi}{\partial t}, N\right\rangle+\left\langle\xi, \frac{\partial N}{\partial t}\right\rangle
$$

Thus

$$
0=\left\langle(G(k) k)^{\prime} \frac{\partial k}{\partial s} N, N\right\rangle+\left\langle\xi, \frac{\partial N}{\partial t}\right\rangle=(G(k) k)^{\prime} \frac{\partial k}{\partial s}+\left\langle\xi, \frac{\partial N}{\partial t}\right\rangle .
$$

From $0 \equiv\left\langle\frac{\partial N}{\partial t}, N\right\rangle$ it follows that there exists $\lambda$ such that

$$
\frac{\partial N}{\partial t}=\lambda \xi
$$

and then combining with the above equality we have

$$
\lambda=-(G(k) k)^{\prime} \frac{\partial k}{\partial s}
$$

and

$$
\frac{\partial N}{\partial t}=-(G(k) k)^{\prime} \frac{\partial k}{\partial s} \xi
$$

v) Since $\xi=(\cos \theta, \sin \theta)$, then $N=(-\sin \theta, \cos \theta)$, we obtain

$$
\frac{\partial \xi}{\partial t}=(-\sin \theta, \cos \theta) \frac{\partial \theta}{\partial t}=\frac{\partial \theta}{\partial t} N
$$

and comparing it with (iv) we conclude that

$$
\frac{\partial \theta}{\partial t}=(G(k) k)^{\prime} \frac{\partial k}{\partial s}
$$

In other cases,

$$
\frac{\partial \xi}{\partial s}=(-\sin \theta, \cos \theta) \frac{\partial \theta}{\partial s}=\frac{\partial \theta}{\partial s} N
$$


It follows from $\frac{\partial \xi}{\partial s}=k N$ that

$$
\frac{\partial \theta}{\partial s}=k
$$

vi) Consider the closed curve $\gamma=\left\{(x(u), y(u)) \mid u \in \mathbb{S}^{1}\right\}$ in $\mathbb{R}^{2}$. Then it is well known that the area of the domain by the curve $\gamma$ can be expressed by the formula

$$
A=\frac{1}{2} \int_{0}^{2 \pi}\left(x \frac{\partial y}{\partial u}-y \frac{\partial x}{\partial u}\right) d u=-\frac{1}{2} \int_{0}^{2 \pi}\langle\gamma(u), v N\rangle d u .
$$

Then

$$
\frac{d A}{d t}=-\frac{1}{2} \int_{0}^{2 \pi}\left\langle\frac{\partial \gamma}{\partial t}, v N\right\rangle d u-\frac{1}{2} \int_{0}^{2 \pi}\left\langle\gamma, \frac{\partial v}{\partial t} N\right\rangle d u-\frac{1}{2} \int_{0}^{2 \pi}\left\langle\gamma, \frac{\partial N}{\partial t} v\right\rangle d u .
$$

By (1.3) and (i), (iv) we obtain

$$
\begin{aligned}
\frac{d A}{d t}= & -\frac{1}{2} \int_{0}^{2 \pi}\langle G(k) k N, v N\rangle d u+\frac{1}{2} \int_{0}^{2 \pi}\left\langle\gamma, G(k) k^{2} v N\right\rangle d u+\frac{1}{2} \int_{0}^{2 \pi}\left\langle\gamma, \frac{\partial}{\partial s}(G(k) k) v \xi\right\rangle d u \\
= & -\frac{1}{2} \int_{0}^{2 \pi} G(k) k v d u+\frac{1}{2} \int_{0}^{2 \pi}\left\langle\gamma, G(k) k^{2} N\right\rangle v d u+\frac{1}{2} \int_{0}^{2 \pi}\left\langle\gamma, \frac{\partial}{\partial u}(G(k) k) \xi\right\rangle d u \\
=- & \frac{1}{2} \int_{0}^{2 \pi} G(k) k v d u+\frac{1}{2} \int_{0}^{2 \pi}\left\langle\gamma, G(k) k^{2} N\right\rangle v d u \\
& \quad+\frac{1}{2} \int_{0}^{2 \pi}\left\langle\gamma, \frac{\partial}{\partial u}(G(k) k \xi)\right\rangle d u-\frac{1}{2} \int_{0}^{2 \pi}\left\langle\gamma, G(k) k \frac{\partial \xi}{\partial u}\right\rangle d u .
\end{aligned}
$$

Thus from $\frac{\partial \xi}{\partial w}=v k N$ we arrive at

$$
\begin{aligned}
\frac{d A}{d t}= & -\frac{1}{2} \int_{0}^{2 \pi} G(k) k v d u+\frac{1}{2} \int_{0}^{2 \pi}\left\langle\gamma, G(k) k^{2} N\right\rangle v d u \\
& \quad-\frac{1}{2} \int_{0}^{2 \pi}\left\langle\frac{\partial \gamma}{\partial u}, G(k) k \xi\right\rangle d u-\frac{1}{2} \int_{0}^{2 \pi}\left\langle\gamma, G(k) k^{2} N\right\rangle v d u \\
= & -\frac{1}{2} \int_{0}^{2 \pi} G(k) k v d u-\frac{1}{2} \int_{0}^{2 \pi}\langle k \xi, G(k) k \xi\rangle v d u \\
= & -\frac{1}{2} \int_{0}^{2 \pi} G(k) k v d u-\frac{1}{2} \int_{0}^{2 \pi} G(k) k v d u \\
= & -\int_{0}^{2 \pi} G(k) k v d u \\
= & -\int_{0}^{L} G(k) k d s .
\end{aligned}
$$

vii) By (iii) and (v) we drive the following equation

$$
\frac{\partial k}{\partial t}=\frac{\partial}{\partial t} \frac{\partial \theta}{\partial s}=\frac{\partial}{\partial s} \frac{\partial \theta}{\partial t}+G(k) k^{2} \frac{\partial \theta}{\partial s}=\frac{\partial}{\partial s}\left((G(k) k)^{\prime} \frac{\partial k}{\partial s}\right)+G(k) k^{3} .
$$

Thus, the proof of Lemma 2.1 is completed.

Similarly to [2], we can use the angle $\theta$ of the tangent line as the parameter of the curve and then write the curvature $k=k(\theta)$ in terms of this parameter which is $2 \pi$ periodic curvature function of convex curve. The following results gives the necessary and sufficient condition for 
some one-parameter function as the curvature function of a simple closed curve(cf. Lemma 4.1.1 in $[2])$.

Lemma 2.2. A positive $2 \pi$ periodic function represents the curvature function of a closed and strictly convex $C^{2}$ curves in the plane if and only if

$$
\int_{0}^{2 \pi} \frac{\cos \theta}{k(\theta)} d \theta=\int_{0}^{2 \pi} \frac{\sin \theta}{k(\theta)} d \theta=0 .
$$

According to the flow (1.3), we take $\tau=t$ as the time parameter and use $\theta$ as other coordinate and hence change variables from $(u, t)$ to $(\theta, \tau)$.

\section{Lemma 2.3.}

$$
\begin{aligned}
\frac{\partial k}{\partial \tau} & =k^{2}\left(\frac{\partial^{2}}{\partial \theta^{2}}(G(k) k)+G(k) k\right) \\
& =k^{2}(G(k) k)^{\prime} \frac{\partial^{2} k}{\partial \theta^{2}}+k^{2}(G(k) k)^{\prime \prime}\left(\frac{\partial k}{\partial \theta}\right)^{2}+G(k) k^{3}
\end{aligned}
$$

Proof. By the chain rule and Lemma 2.1 (iii),(v), we get

$$
\begin{aligned}
\frac{\partial k}{\partial t} & =\frac{\partial k}{\partial \tau}+\frac{\partial k}{\partial \theta} \frac{\partial \theta}{\partial t} \\
& =\frac{\partial k}{\partial \tau}+\frac{\partial k}{\partial \theta}(G(k) k)^{\prime} \frac{\partial k}{\partial s} \\
& =\frac{\partial k}{\partial \tau}+\frac{\partial k}{\partial \theta}(G(k) k)^{\prime} \frac{\partial k}{\partial \theta} \frac{\partial \theta}{\partial s} \\
& =\frac{\partial k}{\partial \tau}+(G(k) k)^{\prime} k\left(\frac{\partial k}{\partial \theta}\right)^{2}
\end{aligned}
$$

On the other hand, from Lemma 2.1 (vii) we obtain

$$
\begin{aligned}
\frac{\partial k}{\partial t} & =\frac{\partial}{\partial s}\left((G(k) k)^{\prime} \frac{\partial k}{\partial s}\right)+G(k) k^{3} \\
& =\frac{\partial \theta}{\partial s} \frac{\partial}{\partial \theta}\left((G(k) k)^{\prime} \frac{\partial k}{\partial \theta} \frac{\partial \theta}{\partial s}\right)+G(k) k^{3} \\
& =k \frac{\partial}{\partial \theta}\left((G(k) k)^{\prime} \frac{\partial k}{\partial \theta} k\right)+G(k) k^{3} \\
& =k \frac{\partial}{\partial \theta}\left(k \frac{\partial}{\partial \theta}(G(k) k)\right)+G(k) k^{3} \\
& =k^{2} \frac{\partial^{2}}{\partial \theta^{2}}(G(k) k)+(G(k) k)^{\prime} k\left(\frac{\partial k}{\partial \theta}\right)^{2}+G(k) k^{3} .
\end{aligned}
$$

By comparing the above two equalities we have the desired results.

Throughout this paper, we will deal with this equation and replace $\tau$ by $t$.

Lemma 2.4. Suppose (H1) hold. Then the general curve shortening problem (1.3) for convex curves is equivalent to the cauchy problem

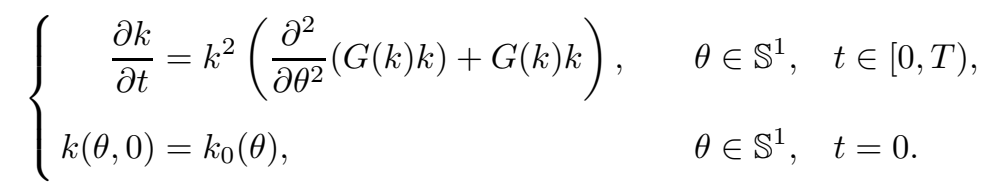


where $0<\alpha<1, k \in C^{2+\alpha, 1+\frac{\alpha}{2}}\left(\mathbb{S}^{1} \times(0, T)\right), k_{0}(\theta)$ is the curvature function of the initial curve $\gamma_{0}(\theta)$.

Proof. If $\gamma(\cdot, t)$ are the classical solution of problem (1.3). Then by Lemma 2.3 the curvature function, expressed in $\theta$ coordinates, satisfies (2.2).

If $k_{0}(\theta)$ is the curvature function for the curve $\gamma_{0}(\theta)$ and $k(\theta, t)$ satisfies (2.2). Then for each $t \geq 0$, we can define the curves by the formula

$$
x(\theta, t)=\int_{0}^{\theta} \frac{\cos u}{k(u, t)} d u, \quad y(\theta, t)=\int_{0}^{\theta} \frac{\sin u}{k(u, t)} d u .
$$

Let $\gamma(\theta, t)=(x(\theta, t), y(\theta, t))$, then $\zeta=(\cos \theta, \sin \theta)$ and $N=(-\sin \theta, \cos \theta)$ are respectively the tangent vector and the inward normal vector of the curve $\gamma(\cdot, t)$. Combining (2.2) with (2.3) we have

$$
\begin{aligned}
\frac{\partial x}{\partial t} & =-\int_{0}^{\theta} \frac{\cos u}{k^{2}} \frac{\partial k}{\partial t} d u \\
& =-\int_{0}^{\theta} \cos u\left(\frac{\partial^{2}}{\partial u^{2}}(G(k) k)+G(k) k\right) d u \\
& =-\int_{0}^{\theta} \cos u \frac{\partial^{2}}{\partial u^{2}}(G(k) k) d u-\int_{0}^{\theta} \cos u G(k) k d u .
\end{aligned}
$$

So that

$$
\begin{aligned}
\frac{\partial x}{\partial t} & =-\int_{0}^{\theta} \sin u \frac{\partial}{\partial u}(G(k) k) d u-\int_{0}^{\theta} \cos u G(k) k d u-\cos \theta \frac{\partial}{\partial \theta}(G(k) k)+\left.\frac{\partial}{\partial \theta}(G(k) k)\right|_{\theta=0} \\
& =\int_{0}^{\theta} \cos u G(k) k d u-G(k) k \sin \theta-\int_{0}^{\theta} \cos u G(k) k d u-\cos \theta \frac{\partial}{\partial \theta}(G(k) k)+\left.\frac{\partial}{\partial \theta}(G(k) k)\right|_{\theta=0} \\
& =-G(k) k \sin \theta-\cos \theta \frac{\partial}{\partial \theta}(G(k) k)+\left.\frac{\partial}{\partial \theta}(G(k) k)\right|_{\theta=0} .
\end{aligned}
$$

For the same reason the following equality holds

$$
\frac{\partial y}{\partial t}=G(k) k \cos \theta-\sin \theta \frac{\partial}{\partial \theta}(G(k) k)-\left.G(k) k\right|_{\theta=0} .
$$

By setting $\theta=2 \pi$ to the above two equalities we obtain $\frac{\partial x}{\partial t}=0, \frac{\partial y}{\partial t}=0$. On the other hand, $x(2 \pi, 0)=0, y(2 \pi, 0)=0$. Because $\gamma_{0}$ is the closed curve in the plane, then applying Lemma 2.2 we obtain $x(2 \pi, 0)=0, y(2 \pi, 0)=0$, and

$$
\int_{0}^{2 \pi} \frac{\cos \theta}{k(\theta, t)} d \theta=\int_{0}^{2 \pi} \frac{\sin \theta}{k(\theta, t)} d \theta=0 .
$$

By applying Lemma 2.2 again, the curve $\gamma(\theta, t)$ which is defined by (2.3) is closed and then we have

$$
\frac{\partial \gamma}{\partial t}(\theta, t)=G(k) k N-\frac{\partial}{\partial \theta}(G(k) k) \xi-(a(0, t), b(0, t))
$$

where

$$
(a(0, t), b(0, t))=\left(-\left.\frac{\partial}{\partial \theta}(G(k) k)\right|_{\theta=0},\left.G(k) k\right|_{\theta=0}\right) .
$$


Set

$$
\begin{gathered}
\theta=\theta(u, \tau), \quad t=\tau, \\
\hat{\gamma}(u, \tau)=\gamma(\theta(u, \tau), \tau)+\left(\int_{0}^{\tau} a(0, t) d t, \int_{0}^{\tau} b(0, t) d t\right),
\end{gathered}
$$

where $\theta=\theta(u, \tau)$ is the unique solution of the following ordinary equation

$$
\left\{\begin{array}{l}
\frac{\partial \theta}{\partial \tau}=k \frac{\partial}{\partial \theta}(G(k) k), \quad \tau \in[0, T), \\
\theta(u, 0)=u
\end{array}\right.
$$

Combining (2.4) with(2.5) we know that $\hat{\gamma}(u, \tau)$ satisfies

$$
\frac{\partial \hat{\gamma}}{\partial \tau}=G(k) k N
$$

and hence we obtain the general curve shortening flow (1.3).

Using the standard results on parabolic equations (cf. [8]) we obtain the existence results of the problem (2.2).

Lemma 2.5. Suppose $k_{0}(\theta) \in C\left(\mathbb{S}^{1}\right)$ and $(H 1)$ holds. Then there exists

$$
T>0, \quad k \in C^{2,1}\left(\mathbb{S}^{1} \times(0, T)\right) \cap C\left(\mathbb{S}^{1} \times[0, T)\right),
$$

satisfying (2.2).

By maximum principle it shows that if the initial curve are strictly convex, then the curves remain so under the flow (1.3).

Lemma 2.6. Suppose (H1) holds and $k(\theta, t)$ is the classical solution of (2.2). If $k_{0}(\theta)$ is positive on $\mathbb{S}^{1}$, then $k_{\min }(t)=\inf \left\{k(\theta, t) \mid \theta \in \mathbb{S}^{1}\right\}$ is a nondecreasing function.

Proof. By contradiction, there exist $0 \leq t_{1}^{\prime} \leq t_{1}<T$ such that $k_{\min }(t)$ is nondecreasing in $\left[0, t_{1}^{\prime}\right]$ and $k_{\min }\left(t_{1}^{\prime}\right)>k_{\min }\left(t_{1}\right)>0$. We suppose $t_{1}^{\prime}=0$ without losing the generalization. Set

$$
t_{0}=\inf \left\{t \in\left[0, t_{1}\right] \mid k_{\min }(t) \leq k_{\min }\left(t_{1}\right)\right\} .
$$

By the regularity of $k(\theta, t)$ we know that there is $\theta_{0} \in \mathbb{S}^{1}$ such that $k_{\min }\left(t_{0}\right)=k\left(\theta_{0}, t_{0}\right)$. It is easy to see that $k_{\min }\left(t_{0}\right)>0$ and then we have

$$
\frac{\partial^{2} k}{\partial \theta^{2}}\left(\theta_{0}, t_{0}\right) \geq 0, \quad \frac{\partial k}{\partial \theta}\left(\theta_{0}, t_{0}\right)=0, \quad k\left(\theta_{0}, t_{0}\right)>0 .
$$

Hence from (2.2) and $(H 1)$ this yields

$$
\left.\frac{\partial k}{\partial t}\left(\theta_{0}, t\right)\right|_{t=t_{0}}>0
$$

and it contradicts to the hypothesis of $t_{0}$. So that we obtain the desired results.

Some further consequences of Lemmas 2.4 2.6 are part of Proposition 1.1] 
Corollary 2.7. Suppose (H1) holds. Then there exist $T>0$ and the unique $\gamma(u, t) \in C^{2,1}\left(\mathbb{S}^{1} \times\right.$ $(0, T)) \cap C\left(\mathbb{S}^{1} \times[0, T)\right)$ satisfying the generalized curve shortening flow (1.3).

According to the flow (1.3) we consider the support function (cf. [9], [10]) of $\gamma(u, t)$ by defining

$$
h(\theta, t)=\langle\gamma(u(\theta, t), t),(\sin \theta,-\cos \theta)\rangle, \quad \theta \in[0,2 \pi],
$$

where $\theta$ is the tangent angle of $\gamma(\cdot, t)$ and the unit normal vector $N=-(\sin \theta,-\cos \theta)$.

Applying the equation (1.3) we see that

$$
\frac{\partial h}{\partial t}=\left\langle\frac{\partial \gamma}{\partial t}+\frac{\partial u}{\partial t} \frac{\partial \gamma}{\partial u},-N\right\rangle=\left\langle G(k) k N+\frac{\partial u}{\partial t}\left|\frac{\partial \gamma}{\partial u}\right| \xi,-N\right\rangle=-G(k) k .
$$

Using the methods in [10], we can compute the inward curvature of $\gamma(\cdot, t)$ by the formula

$$
k=\left(\frac{\partial^{2} h}{\partial \theta^{2}}+h\right)^{-1}
$$

Then $h(\theta, t)$ satisfies the following equation

$$
\frac{\partial h}{\partial t}\left(\frac{\partial^{2} h}{\partial \theta^{2}}+h\right) G^{-1}\left(\left(\frac{\partial^{2} h}{\partial \theta^{2}}+h\right)^{-1}\right)=-1 .
$$

By making use of the maximum principle, we can obtain the containment principle of the flow (1.3) (cf. [Z]).

Lemma 2.8. Let $\gamma_{1}$ and $\gamma_{2}: \mathbb{S}^{1} \times[0, T)$ be two classical solutions of the flow (1.3)). If $\gamma_{2}(\cdot, 0)$ is in the domain enclosed by $\gamma_{1}(\cdot, 0)$, then $\gamma_{2}(\cdot, t)$ is contained in the domain enclosed by $\gamma_{2}(\cdot, t)$ for all $t \in[0, T)$.

Proof. Set $h_{1}(\theta, t)$ and $h_{2}(\theta, t)$ be the support functions of $\gamma_{1}(\cdot, t)$ and $\gamma_{2}(\cdot, t)$. Then $h_{1}(\theta, t)$ and $h_{2}(\theta, t)$ satisfy the equations (2.6). Because $\gamma_{2}(\cdot, 0)$ is in the domain enclosed by $\gamma_{1}(\cdot, 0)$ we can select $h_{1}(\theta, 0)$ and $h_{2}(\theta, 0)$ such that $h_{1}(\theta, 0) \geq h_{2}(\theta, 0)$ for $\theta \in \mathbb{S}^{1}$. Thus by applying the maximum principle of parabolic equations, we deduce that $h_{1}(\theta, t) \geq h_{2}(\theta, t)$ for all $t \in[0, T)$ and then we obtain the desired results.

In order to prove some isometric inequalities we need the following lemma which belongs to Ben.Andrews (cf, Lemma II 3.3 in [3]).

Lemma 2.9. Let $M$ be a compact manifold with a volume form $d \mu$, and let $\xi$ be a continuous function on $M$. Then for any non-decreasing function $F$, there holds

$$
\frac{\int_{M} \xi F(\xi) d \mu}{\int_{M} F(\xi) d \mu} \geq \frac{\int_{M} \xi d \mu}{\int_{M} d \mu} .
$$

The next two lemmas roughly characterized the behavior of the geometric quantity when $\gamma(\cdot, t)$ is contracting to a point under the flow (1.3). 
Lemma 2.10. Suppose (H1) hold. Then under the flow (1.3) we have

$$
\frac{d}{d t}\left(\frac{L^{2}}{A}\right) \leq 0
$$

Proof. By Lemma 2.1 (ii) and (vi), i.e,

$$
\begin{gathered}
\frac{d L}{d t}=-\int_{0}^{L} G(k) k^{2} d s=-\int_{0}^{2 \pi} G(k) k d \theta, \\
\frac{d A}{d t}=-\int_{0}^{L} G(k) k d s=-\int_{0}^{2 \pi} G(k) d \theta,
\end{gathered}
$$

we obtain

$$
\frac{d}{d t}\left(\frac{L^{2}}{A}\right)=-\frac{2 L}{A}\left(\int_{0}^{2 \pi} G(k) k d \theta-\frac{L}{2 A} \int_{0}^{2 \pi} G(k) d \theta\right) .
$$

From the isometric inequality in $[\mathrm{O}]$ the following inequality holds for convex curves,

$$
\frac{\pi L}{A} \leq \int_{0}^{L} k^{2} d s=\int_{0}^{2 \pi} k d \theta,
$$

Substituting (2.8) into (2.7) we have

$$
\begin{aligned}
\frac{d}{d t}\left(\frac{L^{2}}{A}\right) & \leq-\frac{2 L}{A}\left(\int_{0}^{2 \pi} G(k) k d \theta-\frac{1}{2 \pi} \int_{0}^{2 \pi} G(k) d \theta \int_{0}^{2 \pi} k d \theta\right) \\
& =-\frac{2 L}{A} \int_{0}^{2 \pi} G(k) d \theta\left(\frac{\int_{0}^{2 \pi} G(k) k d \theta}{\int_{0}^{2 \pi} G(k) d \theta}-\frac{\int_{0}^{2 \pi} k d \theta}{\int_{0}^{2 \pi} 1 d \theta}\right) .
\end{aligned}
$$

Setting $M=\mathbb{S}^{1}, \psi=k, d \mu=d \theta$ in Lemma 2.9 one can show that

$$
\frac{\int_{0}^{2 \pi} G(k) k d \theta}{\int_{0}^{2 \pi} G(k) d \theta} \geq \frac{\int_{0}^{2 \pi} k d \theta}{\int_{0}^{2 \pi} d \theta} .
$$

So that the proof is completed by means of (2.9) and (2.10).

Lemma 2.11. Suppose (H1) holds. Under the flow (1.3) if

$$
\lim _{t \rightarrow \omega} A(t)=0
$$

we have

$$
\liminf _{t \rightarrow \omega} L\left(\int_{0}^{L} k^{2} d s-\frac{\pi L}{A}\right) \leq 0 .
$$

Proof. From (2.7) we see that

$$
\begin{aligned}
\frac{d}{d t}\left(\frac{L^{2}}{A}\right) & =-\frac{2 L}{A}\left(\int_{0}^{2 \pi} G(k) k d \theta-\frac{L}{2 A} \int_{0}^{2 \pi} G(k) d \theta\right) \\
& =-\frac{\int_{0}^{2 \pi} G(k) d \theta}{\pi A} \cdot L\left(\frac{2 \pi \int_{0}^{2 \pi} G(k) k d \theta}{\int_{0}^{2 \pi} G(k) d \theta}-\frac{\pi L}{A}\right) .
\end{aligned}
$$

Applying

$$
\frac{d A}{d t}=-\int_{0}^{2 \pi} G(k) d \theta
$$


to (2.12) we conclude that

$$
\frac{d}{d t}\left(\frac{L^{2}}{A}\right)=\frac{1}{\pi} \frac{d}{d t}(\ln A) \cdot L\left(\frac{2 \pi \int_{0}^{2 \pi} G(k) k d \theta}{\int_{0}^{2 \pi} G(k) d \theta}-\frac{\pi L}{A}\right) .
$$

Using (2.10) we have

$$
\frac{d}{d t}\left(\frac{L^{2}}{A}\right) \leq \frac{1}{\pi} \frac{d}{d t}(\ln A) \cdot L\left(\int_{0}^{2 \pi} k d \theta-\frac{\pi L}{A}\right) .
$$

Now we prove (2.11) by contradiction. If not, there exists $\delta>0$ such that if $\lim _{t \rightarrow \omega} A(t)=0$ then

$$
\liminf _{t \rightarrow \omega} L\left(\int_{0}^{L} k^{2} d s-\frac{\pi L}{A}\right) \geq 2 \delta .
$$

Hence there exists $\beta=\beta(\delta) \in(0, \omega)$ such that if $t \in(\beta, \omega)$, then the following inequality holds

$$
L\left(\int_{0}^{L} k^{2} d s-\frac{\pi L}{A}\right) \geq \delta .
$$

From (2.13) and (2.14) we obtain

$$
\frac{d}{d t}\left(\frac{L^{2}}{A}\right) \leq \frac{\delta}{\pi} \frac{d}{d t}(\ln A), \quad t \in(\beta, \omega) .
$$

Integrating from $\beta$ to $t$ we have

$$
\begin{gathered}
\frac{L^{2}}{A}(t)-\frac{L^{2}}{A}(\beta) \leq \frac{\delta}{\pi}(\ln A(t)-\ln A(\beta)), \\
-\frac{L^{2}}{A}(\beta) \leq \frac{\delta}{\pi}(\ln A(t)-\ln A(\beta)) .
\end{gathered}
$$

Using $\lim _{t \rightarrow \omega} A(t)=0$ one easily verifies that

$$
-\frac{L^{2}(\beta)}{A(\beta)}+\frac{\delta}{\pi} \ln A(\beta)=-\infty
$$

and this contradicts to Proposition 1.1 and then the proof of Lemma 2.11 is completed.

\section{ASYMPTOTIC BEHAVIOR}

In this section we will study the asymptotic behavior of the curvature under the flow (1.3) and prove the main theorem of this paper.

We recall the following two auxiliary results, which belong to M.E.Gage [14], [15] and R.Osserman [16].

\section{Lemma 3.1. (M.E.Gage)}

(a) There is a non-negative functional $F(\gamma)$ which is defined for all $C^{2}$ convex curves and satisfies

$$
(1-F(\gamma)) \int_{0}^{L} k^{2} d s-\frac{\pi L}{A} \geq 0 .
$$

(b) Given a sequence of convex curves $\left\{\gamma_{i}\right\}$ such that $\lim _{i \rightarrow \infty} F\left(\gamma_{i}\right)=0$. If these normalized curves $\eta_{i}=\sqrt{\frac{\pi}{A}} \gamma_{i}$ lie in a fixed bounded region of the plane, then the domain $H_{i}$ which enclosed by $\eta_{i}$ converges to the disk in the Hausdorff metric.

(c) $F(\gamma)=0$ if and only if $\gamma$ is a circle. 
Lemma 3.2. (Bonneson inequality) Let $\gamma$ be a $C^{1}$ closed convex curve. Then

$$
\frac{L^{2}}{A}-4 \pi \geq \frac{\pi^{2}}{A}\left(r_{\text {out }}-r_{\text {in }}\right)^{2}
$$

Definition 3.3. Let $A, B$ be two closed convex sets and $A_{\epsilon}=\left\{x \in \mathbb{R}^{2} \mid \operatorname{dist}(x, A) \leq \epsilon\right\}$. Then the Hausdorff distance between the sets $A$ and $B$ is given by

$$
d_{H}(A, B)=\inf \left\{\epsilon \mid A \subseteq B_{\epsilon}, B \subseteq A_{\epsilon}\right\} .
$$

Proof of Theorem 1.2 (i). We use the idea in [15]. Consider the geometric quantities $L(t)$ and $A(t)$ according to the flow $\gamma(\cdot, t)$ satisfying (1.3). By (3.1) we have

$$
\int_{0}^{L(t)} k^{2}(\theta, t) d s-\frac{\pi L(t)}{A(t)} \geq F(\gamma(t)) \int_{0}^{L(t)} k^{2}(\theta, t) d s .
$$

Using Schwartz inequality we see that

$$
(2 \pi)^{2}=\left(\int_{0}^{L(t)} k(\theta, t) d s\right)^{2} \leq \int_{0}^{L(t)} k^{2}(\theta, t) d s \int_{0}^{L(t)} 1 d s=L(t) \int_{0}^{L(t)} k^{2}(\theta, t) d s .
$$

Substituting (3.4) into (3.3) we obtain

$$
L(t)\left(\int_{0}^{L(t)} k^{2}(\theta, t) d s-\frac{\pi L(t)}{A(t)}\right) \geq F(\gamma) L(t) \int_{0}^{L(t)} k^{2}(\theta, t) d s \geq 4 \pi^{2} F(\gamma(t)) .
$$

In the following steps we will show that if $\forall t_{i} \in[0, \omega)$ which satisfies $\lim _{i \rightarrow+\infty} t_{i}=\omega$ then the limitations in Theorem 1.2 (i),(ii) hold.

By substituting $\gamma_{i}=\gamma\left(\cdot, t_{i}\right), A\left(t_{i}\right), L\left(t_{i}\right)$ into (3.5) and using (2.11) we have

$$
\lim _{i \rightarrow+\infty} F\left(\gamma_{i}\right)=0
$$

Next we show that the normalized curve $\eta_{i}=\sqrt{\frac{\pi}{A}} \gamma_{i}$ lies in a bound region. From Lemma 2.10, we observe that $\frac{L^{2}}{A}$ decreases under under the flow (1.3). One easily verifies that

$$
\frac{L^{2}\left(t_{i}\right)}{A\left(t_{i}\right)}=\frac{L_{n}^{2}\left(t_{i}\right)}{A_{n}\left(t_{i}\right)}
$$

and

$$
\frac{L^{2}\left(t_{i}\right)}{A\left(t_{i}\right)}-4 \pi=\frac{L_{n}^{2}\left(t_{i}\right)}{A_{n}\left(t_{i}\right)}-4 \pi \geq \frac{\pi^{2}}{A_{n}\left(t_{i}\right)}\left(r_{n, \text { out }}\left(t_{i}\right)-r_{n, \text { in }}\left(t_{i}\right)\right)^{2}=\pi\left(r_{n, \text { out }}\left(t_{i}\right)-r_{n, \text { in }}\left(t_{i}\right)\right)^{2},
$$

and $r_{n, i n}\left(t_{i}\right) \leq 1$, by using the Bonneson inequality, where $A_{n}\left(t_{i}\right)$ is the area about the bound domain enclosed by the normalized curve $\eta_{i}$, and $L_{n}\left(t_{i}\right)$ is the perimeter of the curve $\eta_{i}, r_{n, \text { out }}\left(t_{i}\right)$ and $r_{n, i n}\left(t_{i}\right)$ are respectively the radii of the largest and smallest circumscribed circle of the curve $\eta_{i}$. By (3.6) it shows that the outer radii of the normalized curve $\eta_{i}$ are bounded for all $t_{i} \in[0, \omega)$. From Proposition 1.1 we know that $\gamma_{i}$ shrinks to a point under the flow (1.3). Hence if we use $\vartheta$ as the origin in the homothetic expansion of $\mathbb{R}^{2}$, then all of the normalized curve $\eta_{i}$ lies in a ball of radius $2 \mathrm{C}$ around this point. 
Applying Lemma 3.1(b), we see that the sequence of normalized domain $H\left(t_{i}\right)$ according to $\eta_{i}$ converges to the unit disk in the Haudorff metric,

$$
\lim _{i \rightarrow+\infty} H\left(t_{i}\right)=H_{0}
$$

where $H_{0}$ is the unit disk in the plane.

Since L and A are continuous functionals of convex domain then there holds,

$$
\lim _{i \rightarrow+\infty} \frac{L^{2}\left(t_{i}\right)}{A\left(t_{i}\right)}=\lim _{i \rightarrow+\infty} \frac{L_{n}^{2}\left(t_{i}\right)}{A_{n}\left(t_{i}\right)}=\lim _{i \rightarrow+\infty} \frac{L^{2}\left(H\left(t_{i}\right)\right)}{A\left(H\left(t_{i}\right)\right)}=\frac{L^{2}\left(H_{0}\right)}{A\left(H_{0}\right)}=4 \pi .
$$

From (3.2) we have

$$
\frac{L^{2}\left(t_{i}\right)}{A\left(t_{i}\right)}-4 \pi \geq \frac{\pi^{2}}{A\left(t_{i}\right)}\left(r_{\text {out }}\left(t_{i}\right)-r_{\text {in }}\left(t_{i}\right)\right)^{2} \geq \frac{\pi^{2} r_{\text {out }}^{2}\left(t_{i}\right)}{A\left(t_{i}\right)}\left(1-\frac{r_{\text {in }}\left(t_{i}\right)}{r_{\text {out }}\left(t_{i}\right)}\right)^{2} .
$$

It is easy to see that $\pi r_{\text {out }}^{2}\left(t_{i}\right) \geq A\left(t_{i}\right)$, and then

$$
\frac{L^{2}\left(t_{i}\right)}{A\left(t_{i}\right)}-4 \pi \geq\left(1-\frac{r_{i n}\left(t_{i}\right)}{r_{\text {out }}\left(t_{i}\right)}\right)^{2} .
$$

Such that combining this with (3.8) we have

$$
\lim _{i \rightarrow+\infty} \frac{r_{\text {in }}\left(t_{i}\right)}{r_{\text {out }}\left(t_{i}\right)}=1
$$

To prove Theorem 2.1 (ii),(iii), we need the following gradient estimates of the curvature. A similar proof can be found in [11].

Lemma 3.4. Set $\Phi(k)=G(k) k$ and let $k=k(\theta, t)$ be the curvature function of the flow (1.3), where $\theta$ is the tangent angle of the curve $\gamma(\cdot, t)$. Suppose $(H 1)$, (H2) hold and $\varpi \in(0, \omega)$. Then the following inequality holds,

$$
\max _{0 \leq t \leq \varpi, \theta \in \mathbb{S}^{1}}\left|\frac{\partial \Phi}{\partial \theta}\right|^{2} \leq \max \left\{2 \max _{0 \leq t \leq \varpi, \theta \in \mathbb{S}^{1}} \Phi^{2}, \max _{t=0, \theta \in \mathbb{S}^{1}}\left(\left|\frac{\partial \Phi}{\partial \theta}\right|^{2}+2 \Phi^{2}\right)\right\} .
$$

Proof. By Lemma 2.6 we know that $k_{\min }(t)>0$ for $t \in[0, \omega)$. It follows from Lemma 2.3 that $\phi=\Phi(k)$ satisfies the following equation

$$
\frac{\partial \Phi}{\partial t}=k^{2} \Phi^{\prime} \frac{\partial^{2} \Phi}{\partial \theta^{2}}+k^{2} \Phi^{\prime} \Phi
$$

Set

$$
\Psi=\left(\frac{\partial \Phi}{\partial \theta}\right)^{2}+\lambda \Phi^{2},
$$

where $\lambda$ is a constant to be determined. Suppose $\left(\theta_{0}, t_{0}\right) \in \mathbb{S}^{1} \times(0, \varpi]$ such that

$$
\Psi\left(\theta_{0}, t_{0}\right)=\max _{\mathbb{S}^{1} \times[0, \varpi]}\left(\left(\frac{\partial \Phi}{\partial \theta}\right)^{2}+\lambda \Phi^{2}\right) .
$$

Then at $\left(\theta_{0}, t_{0}\right), \Psi$ satisfies the following properties

$$
\frac{\partial \Psi}{\partial \theta}=0, \quad \frac{\partial^{2} \Psi}{\partial \theta^{2}} \leq 0, \quad \frac{\partial \Psi}{\partial t} \geq 0 .
$$


Next we will prove that if selecting some constant $\lambda$ so large, then at $\left(\theta_{0}, t_{0}\right)$ we have

$$
\frac{\partial \Phi}{\partial \theta}=0
$$

Suppose not, then using

$$
0=\frac{\partial \Psi}{\partial \theta}=2 \frac{\partial \Phi}{\partial \theta}\left(\frac{\partial^{2} \Phi}{\partial \theta^{2}}+\lambda \Phi\right)
$$

we see that

$$
0=\frac{\partial^{2} \Phi}{\partial \theta^{2}}+\lambda \Phi
$$

From (3.11) and (3.12) we have

$$
\begin{aligned}
0 \leq & \frac{1}{2} \frac{\partial \Psi}{\partial t} \\
= & \frac{\partial \Phi}{\partial \theta} \frac{\partial^{2} \Phi}{\partial \theta \partial t}+\lambda \Phi \frac{\partial \Phi}{\partial t} \\
= & \frac{\partial}{\partial \theta}\left(\Phi^{\prime} k^{2}\right) \frac{\partial \Phi}{\partial \theta} \frac{\partial^{2} \Phi}{\partial \theta^{2}}+\Phi^{\prime} k^{2} \frac{\partial \Phi}{\partial \theta} \frac{\partial^{3} \Phi}{\partial \theta^{3}}+\frac{\partial}{\partial \theta}\left(\Phi^{\prime} k^{2}\right) \Phi \frac{\partial \Phi}{\partial \theta} \\
& +k^{2} \Phi^{\prime}(k)\left(\frac{\partial \Phi}{\partial \theta}\right)^{2}+\lambda \Phi \Phi^{\prime} k^{2} \frac{\partial^{2} \Phi}{\partial \theta^{2}}+\lambda k^{2} \Phi^{\prime} \Phi^{2} .
\end{aligned}
$$

By $\frac{\partial^{2} \Psi}{\partial \theta^{2}} \leq 0$ we see that

$$
0 \geq \frac{\partial \Phi}{\partial \theta}\left(\frac{\partial^{3} \Phi}{\partial \theta^{3}}+\lambda \frac{\partial \Phi}{\partial \theta}\right) .
$$

Substituting (3.14), (3.16) into 3.15) we obtain

$$
\begin{aligned}
0 \leq-\lambda & \frac{\partial}{\partial \theta}\left(\Phi^{\prime} k^{2}\right) \Phi \frac{\partial \Phi}{\partial \theta}-\lambda k^{2} \Phi^{\prime}\left(\frac{\partial \Phi}{\partial \theta}\right)^{2}+\frac{\partial}{\partial \theta}\left(\Phi^{\prime} k^{2}\right) \Phi \frac{\partial \Phi}{\partial \theta} \\
& +k^{2} \Phi^{\prime}\left(\frac{\partial \Phi}{\partial \theta}\right)^{2}-\lambda^{2} k^{2} \Phi^{2} \Phi^{\prime}+\lambda k^{2} \Phi^{\prime} \Phi^{2} \\
= & (1-\lambda) \frac{\partial}{\partial \theta}\left(\Phi^{\prime} k^{2}\right) \Phi \frac{\partial \Phi}{\partial \theta}+(1-\lambda) \Phi^{\prime} k^{2}\left(\frac{\partial \Phi}{\partial \theta}\right)^{2}+\left(\lambda-\lambda^{2}\right) k^{2} \Phi^{\prime} \Phi^{2} .
\end{aligned}
$$

By the definition of $\Phi$ and $(H 1),(H 2)$, we have

$$
\begin{gathered}
\Phi>0, \quad \Phi^{\prime}>0 \\
\left(\Phi^{\prime} k^{2}\right)^{\prime}=G^{\prime \prime}(k) k^{3}+4 k^{2} G^{\prime}(k)+2 G(k) k=k\left(G(k) k^{2}\right)^{\prime \prime} \geq 0 \\
\frac{\partial}{\partial \theta}\left(\Phi^{\prime} k^{2}\right) \Phi \frac{\partial \Phi}{\partial \theta}=\left(\Phi^{\prime} k^{2}\right)^{\prime}\left(\frac{\partial k}{\partial \theta}\right)^{2} \Phi^{\prime} \Phi \geq 0 \\
\Phi^{\prime} k^{2} \Phi\left(\frac{\partial \Phi}{\partial \theta}\right)^{2}>0, \quad k^{2} \Phi^{\prime} \Phi^{2}>0
\end{gathered}
$$

By selecting $\lambda=2$ and hence substituting it into (3.17) and using (3.18), we obtain the contradiction. Such that (3.13) holds. 
By (3.13) we arrive at

$$
\begin{aligned}
\max _{0 \leq t \leq \varpi, \theta \in \mathbb{S}^{1}}\left|\frac{\partial \Phi}{\partial \theta}\right|^{2} & \leq \max _{\mathbb{S}^{1} \times[0, \varpi]}\left(\left(\frac{\partial \Phi}{\partial \theta}\right)^{2}+\lambda \Phi^{2}\right) \\
& =\Psi\left(\theta_{0}, t_{0}\right) \\
& =\left.2 \Phi^{2}\right|_{\theta=\theta_{0}, t=t_{0}} \\
& \leq \max \left\{2 \max _{0 \leq t \leq \varpi, \theta \in \mathbb{S}^{1}} \Phi^{2}, \max _{t=0, \theta \in \mathbb{S}^{1}}\left(\left|\frac{\partial \Phi}{\partial \theta}\right|^{2}+2 \Phi^{2}\right)\right\} .
\end{aligned}
$$

So that the proof is completed.

Lemma 3.5. Let $q(t)$ be continuous function on $[0, \omega)$. Suppose for each $\varpi \in[0, \omega)$, satisfying

$$
\sup _{0 \leq t \leq \varpi} q(t)<+\infty, \quad \lim _{t \rightarrow \omega} q(t)=+\infty .
$$

Then there exists $\left\{t_{i}\right\} \subset[0, \omega)$, satisfying

$$
\begin{gathered}
\forall i \in\{1,2, \cdots\}, t_{i}<t_{i+1}, \lim _{i \rightarrow+\infty} t_{i}=\omega, \\
q\left(t_{i}\right)=\sup _{0 \leq t \leq t_{i}} q(t) .
\end{gathered}
$$

Proof. Consider the sequence $\left\{t_{i}^{\prime}\right\} \triangleq\left\{T-\frac{T}{i+1}\right\}$. Firstly select $t_{1} \in\left[0, t_{1}^{\prime}\right]$ satisfying

$$
q\left(t_{1}\right)=\sup _{0 \leq t \leq t_{1}^{\prime}} q(t) .
$$

Then

$$
q\left(t_{1}\right)=\sup _{0 \leq t \leq t_{1}} q(t)
$$

It follows from $\lim _{t \rightarrow \omega} q(t)=+\infty$ that we can choose $t_{j_{1}}^{\prime \prime} \in\left\{t_{i}^{\prime}\right\}$ satisfying $q\left(t_{j_{1}}^{\prime \prime}\right)>q\left(t_{1}\right)+2$. So that we can take $t_{2} \in\left[0, t_{j_{1}}^{\prime \prime}\right]$ satisfying

$$
q\left(t_{2}\right)=\sup _{0 \leq t \leq t_{j_{1}}^{\prime \prime}} q(t)
$$

Thus

$$
q\left(t_{2}\right)=\sup _{0 \leq t \leq t_{2}} q(t)
$$

In general we can select $t_{j_{n}}^{\prime \prime} \in\left\{t_{i}^{\prime}\right\}$ satisfying $q\left(t_{j_{n}}^{\prime \prime}\right)>q\left(t_{n}\right)+n+1$ and then choose $t_{n+1} \in\left[0, t_{j_{n}}^{\prime \prime}\right]$ satisfying

$$
q\left(t_{n+1}\right)=\sup _{0 \leq t \leq t_{j_{n}}^{\prime \prime}} q(t)
$$

Then there holds

$$
q\left(t_{n+1}\right)=\sup _{0 \leq t \leq t_{n+1}} q(t)
$$

The desired results follows by taking trace.

Set $q(t)=k_{\max }(t)$. Then by Proposition 1.1 it is easy to verify that $k_{\max }(t)$ satisfies the conditions of Lemma 3.5 
Lemma 3.6. Suppose (H1) and (H2) hold. If we take the sequence $\left\{t_{i}\right\}$ satisfying (3.19) such that the following holds

$$
\forall i \in\{1,2, \cdots\}, \quad k_{\max }\left(t_{i}\right)=\sup _{0 \leq t \leq t_{i}} k_{\max }(t)
$$

and for each $i \in\{1,2, \cdots\}$ there exists $\theta_{i 0} \in \mathrm{S}^{1}$ such that $k_{\max }\left(t_{i}\right)=k\left(\theta_{i 0}, t_{i}\right)$. Then there exist constants $C, C_{1}>0$ depending only on $\gamma_{0}$ such that

$$
\begin{gathered}
\left(1-2\left|\theta-\theta_{i 0}\right|\right) \Phi\left(k_{\max }\left(t_{i}\right)\right) \leq \Phi\left(k\left(\theta, t_{i}\right)\right)+C, \quad \forall \theta \in \mathbb{S}^{1}, \\
\Phi\left(k_{\max }\left(t_{i}\right)\right) \leq C_{1} \Phi\left(k\left(\theta, t_{i}\right)\right), \quad \forall \theta \in \mathbb{S}^{1},
\end{gathered}
$$

where $\Phi(x)=G(x) x$.

Proof. Step 1. Set $\Phi(\theta)=\Phi\left(k\left(\theta, t_{i}\right)\right)$. For each $\theta \in \mathbb{S}^{1}$ by the medium theorem and (3.10) we have

$$
\Phi\left(\theta_{i 0}\right)-\Phi(\theta)=\frac{\partial \Phi}{\partial \theta}(\hat{\theta})\left(\theta_{i 0}-\theta\right) \leq\left(2 \Phi\left(\theta_{i 0}\right)+C\right)\left|\theta_{i 0}-\theta\right| \leq 2 \Phi\left(\theta_{i 0}\right)\left|\theta_{i 0}-\theta\right|+C .
$$

This yields the inequality (3.20).

Step 2. Take $i$ such that $\Phi\left(\theta_{i 0}\right)$ is large sufficiently. Being likely with (3.22), if $\theta_{1}, \theta \in \mathbb{S}^{1}$, we obtain

$$
\Phi\left(\theta_{1}\right)-\Phi(\theta) \leq 2 \Phi\left(\theta_{i 0}\right)\left|\theta_{1}-\theta\right|+C .
$$

It is clear that $\Phi(\theta)=\Phi\left(k\left(\theta, t_{i}\right)\right) \geq \Phi\left(k_{\min }(0)\right)>0$. Set $\theta_{1}=\theta_{i 0}$. If $\left|\theta-\theta_{i 0}\right| \leq \frac{1}{8}$, then from (3.23) we have

$$
\Phi\left(\theta_{i 0}\right)-\Phi(\theta) \leq 2 \Phi\left(\theta_{i 0}\right)\left|\theta_{i 0}-\theta\right|+C \leq \frac{1}{2} \Phi\left(\theta_{i 0}\right)
$$

So that

$$
\frac{1}{2} \Phi\left(\theta_{i 0}\right) \leq \Phi(\theta)
$$

Set $\theta_{1}=\theta_{i 0}+\frac{1}{8}$. Similarly if $\left|\theta-\theta_{1}\right| \leq \frac{1}{16}$, then using (13.23) and $\frac{1}{2} \Phi\left(\theta_{i 0}\right) \leq \Phi\left(\theta_{i 0}+\frac{1}{8}\right)$ we obtain

$$
\frac{1}{4} \Phi\left(\theta_{i 0}\right) \leq \Phi(\theta)
$$

In general, for each $n \in \mathbb{N}$, set

$$
\Lambda_{n}=\left[\theta_{i 0}+\sum_{s=1}^{n} \frac{1}{8 s}, \theta_{i 0}+\sum_{s=1}^{n} \frac{1}{8 s}+\frac{1}{8(n+1)}\right] .
$$

Then if $\theta \in \Lambda_{n}$, such that there holds

$$
\frac{\Phi\left(\theta_{i 0}\right)}{2(n+1)} \leq \Phi(\theta) .
$$

Given $n$ being so large, we may cover $\mathbb{S}^{1}$ by $\Lambda_{1}, \Lambda_{2}, \cdots, \Lambda_{n}$. For each $\theta \in \mathbb{S}^{1}$, we have

$$
\frac{\Phi\left(\theta_{i 0}\right)}{2(n+1)} \leq \Phi(\theta) \text {. }
$$

Taking $C_{1}=2(n+1)$ we have the desired results. 
Set

$$
\hat{k_{\sigma}}\left(t_{i}\right)=\sup \left\{\inf _{[a, b]} k\left(\theta, t_{i}\right) \mid[a, b] \subset(-\infty,+\infty), b-a=\sigma\right\} .
$$

We introduce a lemma of M.E.Gage and R.S.Hamilton (cf. Lemma 5.1 in [2]), which is crucial for studying the asymptotic behavior of the curvature under the general curve shortening flow.

\section{Lemma 3.7.}

$$
\hat{k}_{\sigma}\left(t_{i}\right) r_{i n}\left(t_{i}\right) \leq \frac{1}{1-\Lambda(\sigma)\left(\frac{r_{\text {out }}\left(t_{i}\right)}{r_{\text {in }}\left(t_{i}\right)}-1\right)},
$$

where

$$
\Lambda(\sigma)=\frac{2 \cos \frac{\sigma}{2}}{1-\cos \frac{\sigma}{2}}
$$

Remark 3.8. The proof of Lemma 5.1 in [2] follows only from the convexity of the closed curve $\gamma\left(\cdot, t_{i}\right)$.

Corollary 3.9. Suppose (H1) and (H2) hold. Consider the sequence $\left\{t_{i}\right\}$ satisfying the conditions of Lemma 3.6. Then for the positive $\epsilon$ being small sufficiently, we have

$$
k_{\max }\left(t_{i}\right) r_{i n}\left(t_{i}\right) \leq \frac{2}{1-\epsilon} \cdot \frac{1}{1-\Lambda(\epsilon)\left(\frac{r_{\text {out }}\left(t_{i}\right)}{r_{\text {in }}\left(t_{i}\right)}-1\right)} .
$$

Proof. It follows from (3.20) that

$$
\begin{aligned}
\left(1-2\left|\theta-\theta_{i 0}\right|\right) G\left(k_{\max }\left(t_{i}\right)\right) k_{\max }\left(t_{i}\right) & \leq G\left(k\left(\theta, t_{i}\right)\right) k\left(\theta, t_{i}\right)+C \\
& \leq G\left(k_{\max }\left(t_{i}\right)\right) k\left(\theta, t_{i}\right)+C, \quad \forall \theta \in \mathbb{S}^{1} .
\end{aligned}
$$

So that

$$
\left(1-2\left|\theta-\theta_{i 0}\right|\right) k_{\max }\left(t_{i}\right) \leq k\left(\theta, t_{i}\right)+C, \quad \forall \theta \in \mathbb{S}^{1}
$$

By Proposition 1.1 we have

$$
\lim _{i \rightarrow+\infty} k_{\max }\left(t_{i}\right)=+\infty
$$

Hence from (3.21) and ( $H 1)$ one can easily verify that

$$
\lim _{i \rightarrow+\infty} k\left(\theta, t_{i}\right)=+\infty, \quad \forall \theta \in \mathbb{S}^{1} .
$$

Combining this with (3.25) we obtain

$$
\left(1-2\left|\theta-\theta_{i 0}\right|\right) k_{\max }\left(t_{i}\right) \leq 2 k\left(\theta, t_{i}\right)
$$

for $i$ being large enough, and $\forall \theta \in \mathbb{S}^{1}$. Given any $\epsilon>0$, if $\left|\theta-\theta_{i 0}\right| \leq \frac{\epsilon}{2}$ then

$$
2 k\left(\theta, t_{i}\right) \geq k_{\max }\left(t_{i}\right)(1-\epsilon)
$$

Takes $\sigma=\epsilon$. It follows from the definition of $\hat{k_{\sigma}}\left(t_{i}\right)$ that we have

$$
2 \hat{k}_{\sigma}\left(t_{i}\right) \geq k_{\max }\left(t_{i}\right)(1-\epsilon) .
$$


Then using Lemma 3.7 we obtain

$$
k_{\max }\left(t_{i}\right) r_{i n}\left(t_{i}\right)(1-\epsilon) \leq 2 \hat{k}_{\sigma}\left(t_{i}\right) r_{i n}\left(t_{i}\right) \leq \frac{2}{1-\Lambda(\epsilon)\left(\frac{r_{\text {out }}\left(t_{i}\right)}{r_{\text {in }}\left(t_{i}\right)}-1\right)}
$$

This yields the desired results.

Corollary 3.10. Suppose (H1) and (H2) hold. Consider the sequence $\left\{t_{i}\right\}$ satisfying the conditions of Lemma 3.6. Then for the positive $\epsilon$ being small sufficiently, there exists $i(\epsilon) \in \mathbb{N}$, such that if $i>i(\epsilon)$, we have

$$
k_{\max }\left(t_{i}\right) r_{i n}\left(t_{i}\right) \leq \frac{2}{(1-\epsilon)^{2}}
$$

Proof. By Theorem 1.2 (ii),

$$
\lim _{i \rightarrow+\infty} \frac{r_{\text {in }}\left(t_{i}\right)}{r_{\text {out }}\left(t_{i}\right)}=1
$$

For $i$ being so large we have

$$
1-\Lambda(\epsilon)\left(\frac{r_{\text {out }}\left(t_{i}\right)}{r_{\text {in }}\left(t_{i}\right)}-1\right) \geq 1-\epsilon .
$$

Then substituting (3.26) into (3.24) we obtain the desired results.

Theorem 3.11. Suppose (H1) and (H2) hold. Consider the sequence $\left\{t_{i}\right\}$ satisfying the conditions of Lemma 3.6. Then we have

$$
\lim _{i \rightarrow+\infty} k\left(\theta, t_{i}\right) r_{i n}\left(t_{i}\right)=1, \quad \forall \theta \in \mathbb{S}^{1}
$$

Proof. Set $f_{i}(\theta)=k\left(\theta, t_{i}\right) r_{i n}\left(t_{i}\right)$ and $\Phi(x)=G(x) x$.

Step 1 . We will prove that $f_{i}(\theta)$ is equi-continuous and bounded uniformly.

Because $G(x)$ is non-decreasing function for $x \in(0,+\infty)$. Then by Lemma 3.4 , for $\theta \in \mathbb{S}^{1}$, we arrive at

$$
\begin{aligned}
\left|G\left(k\left(\theta, t_{i}\right)\right) \frac{\partial k}{\partial \theta}\left(\theta, t_{i}\right)\right| & \leq\left|\frac{\partial \Phi}{\partial \theta}\left(\theta, t_{i}\right)\right| \\
& \leq \max _{0 \leq t \leq t_{i}, \theta \in \mathbb{S}^{1}}\left|\frac{\partial \Phi}{\partial \theta}\right| \\
& \leq 2 \max _{0 \leq t \leq t_{i}, \theta \in \mathbb{S}^{1}}|\Phi|+C \\
& =2 \Phi\left(k_{\max }\left(t_{i}\right)\right)+C .
\end{aligned}
$$

So that

$$
\begin{gathered}
\Phi\left(k\left(\theta, t_{i}\right)\right)\left|\frac{\partial k}{\partial \theta}\left(\theta, t_{i}\right)\right|=\left|G\left(k\left(\theta, t_{i}\right)\right) k\left(\theta, t_{i}\right) \frac{\partial k}{\partial \theta}\left(\theta, t_{i}\right)\right| \leq 2 \Phi\left(k_{\max }\left(t_{i}\right)\right) k_{\max }\left(t_{i}\right)+C k_{\max }\left(t_{i}\right), \\
\left|\frac{\partial k}{\partial \theta}\left(\theta, t_{i}\right)\right| \leq 2 \frac{\Phi\left(k_{\max }\left(t_{i}\right)\right)}{\Phi\left(k\left(\theta, t_{i}\right)\right)} k_{\max }\left(t_{i}\right)+C \frac{k_{\max }\left(t_{i}\right)}{\Phi\left(k\left(\theta, t_{i}\right)\right)} \leq 2 \frac{\Phi\left(k_{\max }\left(t_{i}\right)\right)}{\Phi\left(k\left(\theta, t_{i}\right)\right)} k_{\max }\left(t_{i}\right)+C \frac{k_{\max }\left(t_{i}\right)}{\Phi\left(k_{\min }(0)\right)}
\end{gathered}
$$

It follows from (3.25) that

$$
\left|\frac{\partial k}{\partial \theta}\left(\theta, t_{i}\right)\right| \leq(2 C+C) k_{\max }\left(t_{i}\right), \quad \forall \theta \in \mathbb{S}^{1}
$$


By Corollary 3.10 we obtain

$$
\left|\frac{\partial k}{\partial \theta}\left(\theta, t_{i}\right) r_{i n}\left(t_{i}\right)\right| \leq(2 C+C) k_{\max }\left(t_{i}\right) r_{i n}\left(t_{i}\right) \leq C
$$

for $i$ being so large. This yields

$$
\left|\frac{d f_{i}}{d \theta}(\theta)\right| \leq C
$$

On the other hand, by Corollary 3.10 ,

$$
\left|f_{i}(\theta)\right| \leq C
$$

The proof of Step 1 is completed.

Step 2. Because $f_{i}(\theta)$ is equi-continuous and bounded uniformly. Then by Ascoli-Arzela theorem, there exists $f(\theta) \in C\left(\mathbb{S}^{1}\right)$ such that

$$
\lim _{i \rightarrow+\infty} f_{i}(\theta)=f(\theta), \quad \forall \theta \in \mathbb{S}^{1} .
$$

Step 3. We will prove that $f(\theta) \leq 1$ for $\forall \theta \in \mathbb{S}^{1}$.

Suppose the assertion is false. Then there exists $\theta_{0} \in \mathbb{S}^{1}, \beta>0$, such that $f\left(\theta_{0}\right) \geq 1+3 \beta$. Hence there exists also $\delta>0$, such that if $\theta \in\left[\theta_{0}-\delta, \theta_{0}-\delta\right]$, we have

$$
f(\theta) \geq 1+2 \beta .
$$

By (3.28) for $i$ being so large we have

$$
f_{i}(\theta) \geq 1+\beta, \quad \forall \theta \in\left[\theta_{0}-\delta, \theta_{0}-\delta\right]
$$

i.e,

$$
k\left(\theta, t_{i}\right) r_{i n}\left(t_{i}\right) \geq 1+\beta, \quad \forall \theta \in\left[\theta_{0}-\delta, \theta_{0}-\delta\right] .
$$

Take $\sigma=2 \delta$. Then according to the definition of $\hat{k_{\sigma}}\left(t_{i}\right)$ we obtain

$$
1+\beta \leq \hat{k}_{2 \delta}\left(t_{i}\right) r_{\text {in }}\left(t_{i}\right) \leq \frac{1}{1-\Lambda(2 \delta)\left(\frac{r_{\text {out }}\left(t_{i}\right)}{r_{\text {in }}\left(t_{i}\right)}-1\right)} .
$$

Then using

$$
\lim _{i \rightarrow+\infty} \frac{r_{\text {in }}\left(t_{i}\right)}{r_{\text {out }}\left(t_{i}\right)}=1
$$

we have

$$
1+\beta \leq 1
$$

and it is impossible. So $f(\theta) \leq 1$ for $\forall \theta \in \mathbb{S}^{1}$.

Step 4 . We will prove that $f(\theta) \equiv 1$.

By Fatou lemma we have

$$
\int_{0}^{2 \pi} \frac{d \theta}{f(\theta)} \leq \liminf _{i \rightarrow+\infty} \int_{0}^{2 \pi} \frac{d \theta}{f_{i}(\theta)}=\liminf _{i \rightarrow+\infty} \int_{0}^{2 \pi} \frac{d \theta}{k\left(\theta, t_{i}\right) r_{i n}\left(t_{i}\right)}
$$

By the convexity of $\gamma\left(\cdot, t_{i}\right)$, it is easy to verify that

$$
L\left(t_{i}\right)=\int_{0}^{2 \pi} \frac{d \theta}{k\left(\theta, t_{i}\right)}
$$


and substitute it into (3.29) we obtain

$$
\int_{0}^{2 \pi} \frac{d \theta}{f(\theta)} \leq \liminf _{i \rightarrow+\infty} \frac{L\left(t_{i}\right)}{r_{i n}\left(t_{i}\right)}=\liminf _{i \rightarrow+\infty} \frac{L\left(t_{i}\right)}{r_{\text {out }}\left(t_{i}\right)} \cdot \frac{r_{\text {out }}\left(t_{i}\right)}{r_{\text {in }}\left(t_{i}\right)} .
$$

By the geometric property of $r_{\text {out }}$ one can easily verify that $2 \pi r_{\text {out }} \geq L$. Then combining this with (3.30) we have

$$
\int_{0}^{2 \pi} \frac{d \theta}{f(\theta)} \leq \liminf _{i \rightarrow+\infty} \frac{L\left(t_{i}\right)}{r_{i n}\left(t_{i}\right)} \leq 2 \pi \cdot \liminf _{i \rightarrow+\infty} \frac{r_{\text {out }}\left(t_{i}\right)}{r_{\text {in }}\left(t_{i}\right)} .
$$

By making use of Theorem 1.2 (i) again we have

$$
\int_{0}^{2 \pi} \frac{d \theta}{f(\theta)} \leq 2 \pi
$$

On the other hand, by $f(\theta) \leq 1$ in Step 3 we obtain

$$
\int_{0}^{2 \pi} \frac{d \theta}{f(\theta)} \geq 2 \pi
$$

This yields

$$
\int_{0}^{2 \pi} \frac{d \theta}{f(\theta)}=2 \pi
$$

Using $f(\theta) \leq 1$ again we have $f(\theta) \equiv 1$.

Combining (3.28) with Step 4 we completed the proof the theorem.

Remark 3.12. It follows from Cauchy criterion that the following limitation holds

$$
\lim _{t \rightarrow \omega} k(\theta, t) r_{i n}(t)=1, \quad \forall \theta \in \mathbb{S}^{1}
$$

\section{Proof of the theorem 1.2 (ii),(iii).}

Step 1. According to (3.31) we conclude that

$$
\lim _{t \rightarrow \omega} k_{\max }(t) r_{i n}(t)=1, \quad \lim _{t \rightarrow \omega} k_{\min }(t) r_{i n}(t)=1 .
$$

Combining this with Theorem 1.2 (ii) it shows that

$$
\lim _{t \rightarrow \omega} \frac{k_{\max }(t)}{k_{\min }(t)}=1 \text {. }
$$

Step 2. Given $t \in(0, \omega)$ and consider $k_{\max }(t)$. By the property of continuous function, there exists $\theta=\theta(t) \in \mathbb{S}^{1}$ such that $k_{\max }(t)=k(\theta(t), t)$. Then at $(\theta(t), t)$ by the regularity of $k(\theta, t)$ we have

$$
\frac{\partial k}{\partial \theta}=0, \quad \frac{\partial^{2} k}{\partial \theta^{2}} \leq 0, \quad \frac{d k}{d t}=\frac{\partial k}{\partial \theta} \frac{d \theta}{d t}+\frac{\partial k}{\partial t}=\frac{\partial k}{\partial t}
$$

By Lemma 2.3.

$$
\frac{\partial k}{\partial t}=k^{2}\left(\frac{\partial^{2}}{\partial \theta^{2}}(G(k) k)+G(k) k\right) .
$$

Combining (3.33) with (3.34) we see that

$$
\frac{d k_{\max }(t)}{d t} \leq G\left(k_{\max }(t)\right) k_{\max }^{3}(t) .
$$


By the differential inequality and using $k_{\max }(\omega)=+\infty$ we get

$$
\frac{1}{\omega-t} \int_{k_{\max }(t)}^{+\infty} \frac{d x}{G(x) x^{3}} \leq 1 .
$$

From (3.21) it is easy to see that $k_{\min }(\omega)=+\infty$. Similarly we have

$$
\frac{1}{\omega-t} \int_{k_{\min }(t)}^{+\infty} \frac{d x}{G(x) x^{3}} \geq 1 .
$$

Since

$$
\begin{aligned}
\frac{\int_{k_{\min }(t)}^{k_{\max }(t) \frac{d x}{G(x) x^{3}}}}{\int_{k_{\min }(t) \frac{d x}{G(x) x^{3}}}^{+\infty}} & \leq \frac{\frac{k_{\max }(t)-k_{\min }(t)}{G\left(k_{\min }(t)\right) k_{\min }^{3}(t)}}{\frac{1}{2 G\left(k_{\min }(t)\right) k_{\min }^{2}(t)}-\frac{1}{2} \int_{k_{\min }(t)}^{+\infty} \frac{G^{\prime}(x)}{G^{2}(x) x^{2}} d x} \\
& =2\left(\frac{k_{\max }(t)}{k_{\min }(t)}-1\right) \frac{1}{1-G\left(k_{\min }(t)\right) k_{\min }^{2}(t) \int_{k_{\min }(t)}^{+\infty} \frac{G^{\prime}(x)}{G^{2}(x) x^{2}} d x} .
\end{aligned}
$$

We now claim that for the positive $z$ being so large, there holds

$$
1-G(z) z^{2} \int_{z}^{+\infty} \frac{G^{\prime}(x)}{G^{2}(x) x^{2}} d x \geq \frac{2}{2+C_{0}} .
$$

Indeed, by $(H 2)$ we obtain

$$
\int_{z}^{+\infty} \frac{G^{\prime}(x)}{G^{2}(x) x^{2}} d x \leq C_{0} \int_{z}^{+\infty} \frac{d x}{G(x) x^{3}} .
$$

Then

$$
\begin{aligned}
& \int_{z}^{+\infty} \frac{G^{\prime}(x)}{G^{2}(x) x^{2}} d x \leq \frac{C_{0}}{C_{0}+2} \int_{z}^{+\infty} \frac{G^{\prime}(x)}{G(x) x^{2}} d x+\frac{2 C_{0}}{C_{0}+2} \int_{z}^{+\infty} \frac{d x}{G(x) x^{3}}, \\
& \int_{z}^{+\infty} \frac{G^{\prime}(x)}{G^{2}(x) x^{2}} d x \leq \frac{-C_{0}}{C_{0}+2} \int_{z}^{+\infty}\left(\frac{1}{G(x) x^{2}}\right)^{\prime} d x=\frac{C_{0}}{C_{0}+2} \cdot \frac{1}{G(z) z^{2}},
\end{aligned}
$$

and this yields (3.38).

By (3.32), (3.37), (3.38) and applying $k_{\min }(\omega)=+\infty$ we have

$$
\lim _{t \rightarrow \omega} \frac{\int_{k_{\min }(t)}^{k_{\max }(t)} \frac{d x}{G(x) x^{3}}}{\int_{k_{\min }(t)}^{+\infty} \frac{d x}{G(x) x^{3}}}=0 .
$$

Then we obtain

$$
\lim _{t \rightarrow \omega} \frac{\int_{k_{\max }(t)}^{+\infty} \frac{d x}{G(x) x^{3}}}{\int_{k_{\min }(t)}^{+\infty} \frac{d x}{G(x) x^{3}}}=\lim _{t \rightarrow \omega} \frac{\int_{k_{\min }(t)}^{+\infty} \frac{d x}{G(x) x^{3}}-\int_{k_{\min }(t)}^{k_{\max }(t)} \frac{d x}{G(x) x^{3}}}{\int_{k_{\min }(t)}^{+\infty} \frac{d x}{G(x) x^{3}}}=1 .
$$

Combining (3.35), (3.36) with (3.39) we arrive at

$$
\lim _{t \rightarrow \omega} \frac{1}{\omega-t} \int_{k(\theta, t)}^{+\infty} \frac{d x}{G(x) x^{3}}=1, \quad \forall \theta \in \mathbb{S}^{1} .
$$

Acknowledgements. This work is supported by the National Natural Science Foundation of China (10671022) and Doctoral Programme Foundation of Institute of Higher Education of China (20060027023). 


\section{REFERENCES}

[1] I.Bakas and C.Sourdis, Dirichlet sigma models and mean curvature flow, Journal of High Energy Physics, 6 (2007), 1088-1126.

[2] M.E. Gage and R.S. Hamilton, The heat equation shrinking convex plane curves, J. Differential Geometry, 23 (1986), 69-96.

[3] B.Andrews, Evolving convex curves, Calculus of Variations and P.D.E., 7 (1998), 315-371.

[4] E.Calabi, P.J. Olver and A.Tannenbaum, Affine geometry, curve flows and invariant numerical approximations, Adv. Math, 124 (1996), 154-196.

[5] G.Sapiro and A.Tannenbaum, On affine plane curve evolution, J. Functional Analysis, 119 (1994), 79-120.

[6] C.H. Nien and D.H. Tsai, Convex curves moving translationally in the plane, J. Differential equation, 225 (2006), 605-623.

[7] M.Carmo, Differential Geometry of Curves and Surfaces, Beijing: China Machine Press, 2004.

[8] N.V. Krylov, Nonlinear Elliptic and Parabolic Equations of Second Order, D.Reidel, 1987.

[9] R.Schneider, Convex Bodies: The Brunn-Minkowski Theory, Cambridge University Press, 1993.

[10] J.E. Urbas, An expansion of convex hypersurfaces, J. Differential Geometry, 33 (1991), 91-125.

[11] X.P. Zhu, Lectures on mean curvature flows, AMS/IP Studies in Advanced Mathematics, 32, American Mathematical Society, Providence, RI; International Press, Somerville, MA, 2002.

[12] K.S. Chou, Deforming a hypersurface by its Gauss-Kronecker curvature, Comm. Pure. Appl. Math, 38 (1985), $867-882$.

[13] K.S. Chou and X.P. Zhu, The curve shortening problem, Champman Hall/Crc (2000).

[14] M.E. Gage, An isoperimetric inequality with applications to curve shortening, Duke Mathematical Journal, 50 (1984), 1225-1229.

[15] M.E. Gage, Curve shortening makes convex curves circular, Invent. Math, 76 (1984), 357-364.

[16] R.Osserman, Bonnesen-style isoperimetric inequalities, Amer. Math. Monthly, 86 (1979), 1-29.

1. School of Mathematical Sciences, Beijing Normal University, laboratory of Mathematics and Complex Systems, Ministry of Education, Beijing 100875, People’s Republic of China

E-mail address: hrl602@mail.bnu.edu.cn

2. Institute of Mathematics, Fudan University, Shanghai 200433, People's Republic of China

E-mail address: huangronglijane@yahoo.cn

3. Corresponding author. School of Mathematical Sciences, Beijing Normal University, Laboratory of Mathematics and Complex Systems, Ministry of Education, Beijing 100875, People's Republic of China E-mail address: jgbao@bnu.edu.cn 